\title{
A Holistic Approach to the Effects of Fandom: An Application of Self-Expansion Theory
}

\author{
Lanlung Chiang, Aikaterini Manthiou, and Cindy Yunhsin Chou
}

\begin{abstract}
Research on the effect of fans' psychological response to pop-culture destination is arguably limited (Lee et al. 2008). To develop a better understanding of fans' psychological response to pop-culture destination and its relation to their post behaviors, this study expanded self-expansion theory to the existing place attachment framework to examine the relationships between self-expansion, fandom, recollection, place attachment, and post-behavioral intentions. Furthermore, this study argues that place attachment as a key mediates the relationship between fandom and recollection. The survey population were international tourists who are interested in or favor Chinese pop-culture. To represent Chinese pop-culture, particular Chinese pop-stars and their fans were sampled. One-to-one interview method was used to investigate more detailed information from the fans. Nine hundred copies of the questionnaires were used in the survey, and a total of 768 questionnaires were collected during on-site data collection. This study empirically demonstrated that fans and tourists respond to pop-culture tourism emotionally and thus develop further behavioral intentions. This study also examined the bridge created by place attachment and memory recall as it occurs in the cognitive process. The relationship between fandom and post-behavioral intentions is shown.
\end{abstract}

L. Chiang $(\bowtie) \bullet$ C.Y. Chou

Yuan Ze University, Taoyuan City, Taiwan

e-mail: lukech@saturn.yzu.edu.tw; cindy.chou@saturn.yzu.edu.tw

A. Manthiou

NEOMA Business School-Rouen Campus, Mont-Saint-Aignan, France

e-mail: aikaterini.manthiou@neoma-bs.fr 